\title{
Evaluation of Several Computational Methods for the Purpose of Predicting the Structure of a Dinuclear Zinc(II) Complex
}

\author{
Hiroshi SAKIYAMA ${ }^{\mathrm{a} *}$, Akito KAZAMA ${ }^{\mathrm{a}}$, Satoshi SUZUKI ${ }^{\mathrm{b}}$ and Yuzo NISHIDA ${ }^{\mathrm{a}}$

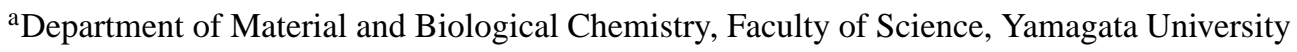 \\ Kojirakawa 1-4-12, Yamagata 990-8560, Japan \\ ${ }^{\mathrm{b}}$ Computer-Aided-Chemistry Laboratory \\ Karasaki 4-203, Kariya, Aichi 448-0039, Japan \\ *e-mail:saki@sci.kj.yamagata-u.ac.jp
}

(Received: July 12, 2007; Accepted for publication: February 4, 2008; Advance publication: February 26, 2008)

The molecular structure of a dinuclear zinc(II) complex was optimized by several computational methods, including ab initio methods, a DFT method, semi-empirical methods, and molecular mechanics methods. The computed structures were compared with a crystallographically obtained structure. The B3LYP/LANL2DZ method could reproduce the crystal structure well, and an axial-elongation tendency around the zinc(II) ion was also reproduced. The resulting structure could be improved by the MP2/3-21G method, indicating the importance of the configuration interaction. Among the semi-empirical methods, only the PM5 and the PM6 could reproduce the crystal structure. For molecular mechanics methods, applicable parameter sets could be determined to reproduce the crystal structure.

Keywords: Structural optimization, Dinuclear zinc(II) complex, Ab initio, DFT, Semi-empirical, Molecular mechanics

\section{Introduction}

Dinuclear zinc structures are often seen in the active sites of metal enzymes, including phosphatases [1, 2], aminopeptidases [3-6], and isoaspartyl dipeptidase [7]. For the purpose of studying the relationship between a structure and a function, we often use synthetic model compounds that contain dinuclear zinc structures similar to the active-site structures. We reported a dinuclear zinc complex $\left.\left[\mathrm{Zn}_{2} \text { (bomp)(OCOMe }\right)_{2}\right] \mathrm{BPh}_{4}$ [bomp $^{-}=$2,6-bis[bis(2-methoxyethyl)aminomethyl $]-4$ methylphenolate] as the first functional model for dinuclear zinc aminopeptidases [8]. The complex hydrolyzes peptide bonds as aminopeptidases do. Although the crystal structure of the complex has been determined, the exact structure during the aminopeptidase-like reaction has not been clarified.

At this stage, if the structure during the reaction could be revealed by a computational method, it would be of great help for understanding the reaction mechanisms. In this study, as a first step for predicting the structures, the optimized structures for the complex cation were obtained using several computational methods in an attempt to find a good method for predicting structures.

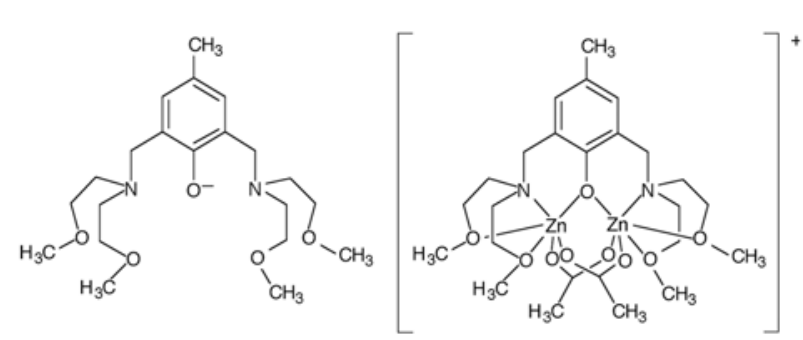

Figure 1. Chemical structures of bomp ${ }^{-}$(left) and $\left[\mathrm{Zn}_{2} \text { (bomp) }(\mathrm{OCOMe})_{2}\right]^{+}$complex cation (right).

\section{Computational methods}

A structure of the complex cation $\left.\left[\mathrm{Zn}_{2} \text { (bomp)(OCOMe }\right)_{2}\right]^{+}$ was optimized using several computational methods, such as ab initio methods, a DFT method, semi-empirical methods, and empirical molecular mechanics (MM) methods. The crystallographically obtained structure was used initially for all computations. An optimization by $a b$ initio methods and DFT was performed using Gaussian 03 software (Gaussian, Inc.) using HF, MP2 [9-13], and B3LYP [14-16] methods with STO-3G, 3-21G, and 
LANL2DZ [17] basis sets. An optimization by semiempirical methods was made using WinMOPAC software (Fujitsu Limited), MOPAC2007 software (Stewart Computational Chemistry) [18], and Winmostar software [19]. The methods used were AM1 [20], PM3 [21], PM5 [22], and PM6 [23]. An optimization by MM2 [24] was performed using Chem3D software (CambridgeSoft Corp.). In this MM2 computation, the parameters around the zinc atom were determined so as to reproduce the crystallographically obtained structure, and the parameter set used is summarized in Appendix 1. An MM optimization based on the MMFF94s force field [25] was performed using CONFLEX software (Conflex Corporation), and the pseudo-potential used is summarized in Appendix 2.

\section{Results and discussion}

The crystal structure of the dinuclear zinc(II) complex $\left.\left[\mathrm{Zn}_{2} \text { (bomp)(OCOMe }\right)_{2}\right] \mathrm{BPh}_{4}$ was previously determined using the single-crystal $\mathrm{X}$-ray method at 297.2 K [8], and the structure of the complex cation $\left[\mathrm{Zn}_{2} \text { (bomp) }(\mathrm{OCOMe})_{2}\right]^{+}$is shown in Figure $2 \mathrm{a}$. Two zinc(II) ions are incorporated in a dinucleating ligand bomp ${ }^{-}$, and the zinc ions are bridged by one phenolic oxygen of the bomp ligand and two acetate ions. The $\mathrm{Zn} \cdots \mathrm{Zn}$ separation is $3.2644(7) \AA$. The coordination geometry around each zinc ion is distorted octahedral. The complex cation has a pseudo $C_{2}$ symmetry, and the twisted angle between an aromatic plane and a plane including two zinc ions and a phenolic oxygen atom is $42^{\circ}$. Another important feature of the complex cation is the elongation of the axial $\mathrm{Zn}-\mathrm{O}$ (ether) bond. The bomp ligand has four ether chelating arms. Two of them are in equatorial positions and the other two are in axial positions. If we define the axial elongation as $100 \times[$ (axial $\mathrm{Zn}-\mathrm{O}$ distance)/(equatorial $\mathrm{Zn}-\mathrm{O}$ distance $)-1]$, the axial elongation was $6.6 \sim 11.0 \%$ for the complex cation.

Computed structures for the complex cation were obtained using several computational methods, such as $a b$ initio methods, DFT methods, semi-empirical methods, and empirical MM methods. The $a b$ initio and DFT computations were made using HF and B3LYP methods with STO-3G, 3-21G, and LANL2DZ basis sets, and the results are summarized in Tables 1-3. When
STO-3G was used, the octahedral coordination geometry around each zinc atom was broken, and the results were not satisfactory. Other basis sets gave successful results, and the B3LYP/LANL2DZ method gave the best result among them. The structure obtained by B3LYP/LANL2DZ is shown in Figure 2b. Deviations from the crystal structure were, at most, $-8 \sim 7 \%$ in the bond lengths of non-hydrogen atoms and $-5 \sim 9 \%$ in the bond angles of non-hydrogen atoms (see Table 1). The $\mathrm{Zn} \cdots \mathrm{Zn}$ separation and the twisted angle were almost reproduced (see Table 2). The computed axial elongation $(4.9 \%)$ was slightly smaller than the crystal structure value (see Table 3), but the elongation tendency was reproduced. The MP2 computations were also performed using the result of B3LYP/LANL2DZ method. The result by MP2/LANL2DZ was not good so much; however, the result by MP2/3-21G could improve the initial structure obtained by the B3LYP/LANL2DZ method (see Tables 1-3). This indicates the importance of the configuration interaction.
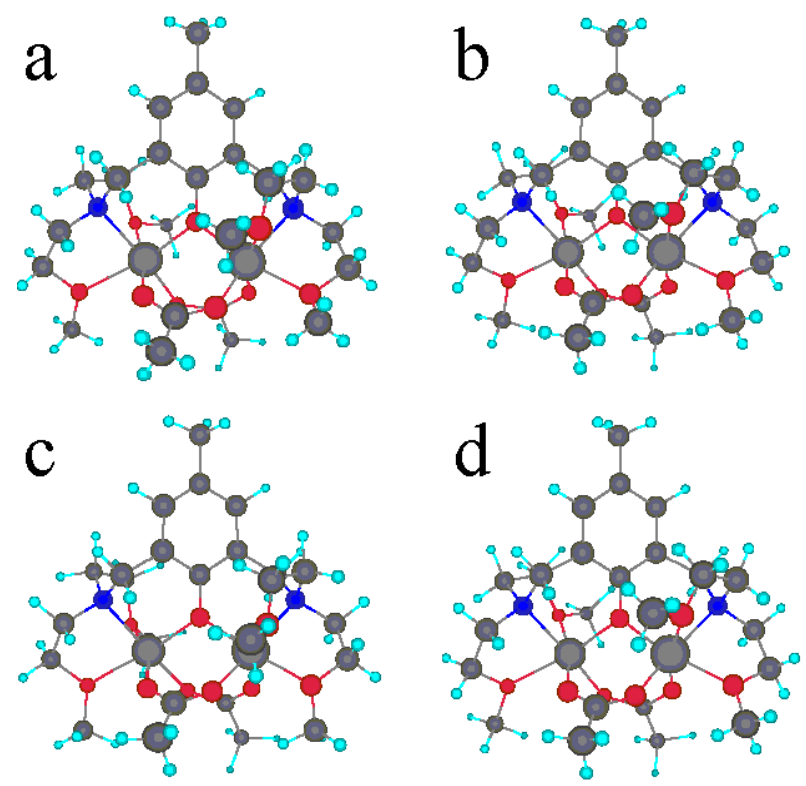

Figure 2. Structures of the $\left.\left[\mathrm{Zn}_{2} \text { (bomp)(OCOMe }\right)_{2}\right]^{+}$ complex cation; crystallographically observed structure (a) and structures computed using the B3LYP/LANL2DZ method (b), the PM5 method (c), and the MMFF94s- $(5,1)$ method (d). 
Table 1. Deviations from the crystal structure for the computed structures.

\begin{tabular}{|c|c|c|c|c|}
\hline \multirow[t]{2}{*}{ Method } & \multicolumn{2}{|c|}{ Deviations in bond distances/\% } & \multicolumn{2}{|c|}{ Deviations in bond angles/\% } \\
\hline & Around zinc & $\begin{array}{l}\text { Non-hydrogen atoms } \\
\text { except for zinc }\end{array}$ & Around zinc & $\begin{array}{l}\text { Non-hydrogen atoms } \\
\text { except for zinc }\end{array}$ \\
\hline HF/STO-3G & failed $^{a}$ & & & \\
\hline $\mathrm{HF} / 3-21 \mathrm{G}$ & $-9 \sim 2$ & $-2 \sim 6$ & $-6 \sim 7$ & $-3 \sim 10$ \\
\hline HF/LANL2DZ & $-8 \sim 6$ & $-2 \sim 6$ & $-5 \sim 9$ & $-5 \sim 10$ \\
\hline B3LYP/STO-3G & failed $^{a}$ & & & \\
\hline B3LYP/3-21G & $-7 \sim 9$ & $-1 \sim 8$ & $-10 \sim 12$ & $-5 \sim 8$ \\
\hline B3LYP/LANL2DZ & $-8 \sim 5$ & $-1 \sim 7$ & $-5 \sim 9$ & $-4 \sim 9$ \\
\hline MP2/3-21G & $-7 \sim 0$ & $0 \sim 9$ & $-6 \sim 6$ & $-5 \sim 5$ \\
\hline MP2/LANL2DZ & $-9 \sim 4$ & $0 \sim 9$ & $-7 \sim 12$ & $-4 \sim 10$ \\
\hline AM1 & failed $^{a}$ & & & \\
\hline PM3 & failed $^{a}$ & & & \\
\hline PM5 & $-9 \sim 3$ & $-2 \sim 4$ & $-10 \sim 15$ & $-6 \sim 9$ \\
\hline PM6 & $-3 \sim 9$ & $-3 \sim 7$ & $-9 \sim 6$ & $-13 \sim 4$ \\
\hline MM2-(6,0) & $-10 \sim 1$ & $-3 \sim 4$ & $-11 \sim 11$ & $-7 \sim 10$ \\
\hline MMFF94s- $(6,0)$ & $-9 \sim 1$ & $-5 \sim 6$ & $-6 \sim 6$ & $-7 \sim 9$ \\
\hline MMFF94s-(0,6) & $-3 \sim 10$ & $-4 \sim 6$ & $-9 \sim 12$ & $-5 \sim 10$ \\
\hline MMFF94s- $(4,2)$ & $-3 \sim 5$ & $-5 \sim 5$ & $-6 \sim 7$ & $-6 \sim 8$ \\
\hline MMFF94s- $(5,1)$ & $-3 \sim 5$ & $-5 \sim 5$ & $-5 \sim 6$ & $-6 \sim 7$ \\
\hline
\end{tabular}

$a$ The coordination geometries around the zinc atoms were broken.

Table 2. Comparison of the crystal structure and the computed structures.

\begin{tabular}{lcc}
\hline Method & Zn $\cdots$ Zn separation/A & $\begin{array}{l}\text { Dihedral angle between the aromatic } \\
\text { ring plane and the Zn-O-Zn plane/ }\end{array}$ \\
\hline X-ray & $3.2644(7)$ & 42 \\
HF/3-21G & 3.323 & 48 \\
HF/LANL2DZ & 3.439 & 48 \\
B3LYP/3-21G & 3.245 & 40 \\
B3LYP/LANL2DZ & 3.450 & 45 \\
MP2/3-21G & 3.258 & 45 \\
MP2/LANL2DZ & 3.449 & 48 \\
PM5 & 3.520 & 45 \\
PM6 & 3.157 & 40 \\
MM2-(6,0) & 3.372 & 45 \\
MMFF94s-(6,0) & 3.396 & 43 \\
MMFF94s-(0,6) & 3.358 & 45 \\
MMFF94s-(4,2) & 3.368 & 41 \\
MMFF94s-(5,1) & 3.375 & 41 \\
\hline
\end{tabular}

Semi-empirical computational optimization was made on the basis of the AM1, PM3, PM5, and PM6 methods. When the structure was optimized using the AM1 or the PM3 method, the octahedral coordination geometry around each zinc atom was broken, and the results were not satisfactory. On the other hand however, the PM5 and the PM6 methods gave successful results; the result obtained by the PM5 method was slightly better, and the structure is shown in Figure 2c. This might have happened because the AM1 and the PM3 method may not be suitable for the metal-ether bonds, whereas the PM5 method may be very suitable. For the PM5 method, devi- ations from the crystal structure were, at most, $-9 \sim 4 \%$ in the bond lengths of non-hydrogen atoms and $-10 \sim 15 \%$ in the bond angles of non-hydrogen atoms. The $\mathrm{Zn} \cdots \mathrm{Zn}$ separation and the twisted angle were close to the crystal structure values. The computed axial elongation was $1.3 \sim 1.4 \%$, smaller than the crystal structure value, but a tendency towards elongation was observed. The quality of the PM5 computation seems to be slightly worse than that using the B3LYP/LANL2DZ method, but the PM5 method was determined to be applicable for zinc complexes of this kind. 
Table 3. Equatorial and axial $\mathrm{Zn}-\mathrm{O}$ (ether) distances and axial elongation. ${ }^{a}$

\begin{tabular}{lccc}
\hline Method & Equatorial Zn-O distance/ $\AA$ & Axial Zn-O distance/A & Axial elongation/\% \\
\hline X-ray & $2.197 \sim 2.215$ & $2.362 \sim 2.439$ & $6.6 \sim 11.0$ \\
HF/3-21G & 2.068 & 2.236 & 8.1 \\
HF/LANL2DZ & 2.154 & $2.259 \sim 2.260$ & 4.9 \\
B3LYP/3-21G & 2.075 & 2.557 & 23.2 \\
B3LYP/LANL2DZ & 2.155 & 2.260 & 4.9 \\
MP2/3-21G & $2.077 \sim 2.086$ & $2.300 \sim 2.310$ & 10.8 \\
MP2/LANL2DZ & 2.135 & 2.227 & 4.3 \\
PM5 & $2.192 \sim 2.193$ & $2.221 \sim 2.222$ & $1.3 \sim 1.4$ \\
PM6 & 2.376 & $2.383 \sim 2.384$ & 0.3 \\
MM2-(6,0) & 2.203 & 2.202 & 0.0 \\
MMFF94s-(6,0) & $2.214 \sim 2.215$ & 2.224 & $0.4 \sim 0.5$ \\
MMFF94s-(0,6) & $2.284 \sim 2.258$ & $2.384 \sim 2.386$ & $4.3 \sim 4.5$ \\
MMFF94s-(4,2) & 2.299 & $2.398 \sim 2.401$ & $4.3 \sim 4.4$ \\
MMFF94s-(5,1) & 2.213 & $2.408 \sim 2.409$ & $8.8 \sim 8.9$ \\
\hline
\end{tabular}

$a$ Axial elongation is defined as $100 \times[($ axial $\mathrm{Zn}-\mathrm{O}$ distance $) /($ equatorial $\mathrm{Zn}-\mathrm{O}$ distance $)-1]$.

In MM computations, parameters around zinc do not exist in general [26]. Thus, in this study, several models were made to reproduce the crystal structure. The first ones were to assume standard zinc-donor bond lengths for all six types of coordination bonds. One was based on the MM2 force field (MM2-(6,0) model) and the other on the MMFF94s force field (MMFF94s-(6,0) model). Parameters additionally defined for both models are summarized in the appendices. The two numbers in parentheses $(n, m)$ indicate that the additional parameters are used for $m$ of the six donor atoms but not used for $n$ of the donor atoms $(0 \leqq m \leqq 6, n=6-m)$. In both resultant structures, the $\mathrm{Zn} \cdot \cdots \mathrm{Zn}$ separation and the twisted angle were good; however, no axial elongation was observed. In a second trial using MMFF94s parameters, no additional parameters were introduced around zinc (MMFF94s-(0,6) model); however, an axial elongation of the order of $4.3 \sim 4.5 \%$ was unexpectedly observed. In this computation, only van der Waals interactions and electrostatic interactions were considered around zinc. Therefore the result may suggest that the zinc-ether bonds are ionic. Thus, in the next model using MMFF94s parameters, the additional pseudo-potentials were introduced for four non-ether donor atoms (MMFF94s-(4,2) model). The obtained structure was really good judging from the deviations of the bond lengths and angles, the $\mathrm{Zn} \cdots \mathrm{Zn}$ separation, and the twist angle. The axial elongation was $4.3 \sim 4.4 \%$. Finally, in a last model using MMFF94s parameters, the additional pseudo-potentials were introduced for four non-ether donor atoms and equatorial ether oxygen atoms (MMFF94s-(5,1) model). This result was the best, and the axial elongation was $8.8 \sim 8.9 \%$, which was quite similar to the crystal structure value. In conclusion, it has been found that the coordination geometry around zinc can be simulated by the molecular mechanics method if we assume ionic zincether bonds.

\section{Conclusion}

The molecular structures of a dinuclear zinc(II) complex were obtained using thirteen computational methods, and the computed structures were evaluated by comparing the non-hydrogen bond lengths, bond angles, $\mathrm{Zn} \cdots \mathrm{Zn}$ separation, twist angle, and axial elongation. It was found that the B3LYP/LANL2DZ method was the best except for the MP2 method. The MP2/3-21G method could improve the structure obtained by B3LYP/LANL2DZ. Among the semi-empirical methods, PM5 was the best; however, it was slightly worse than the B3LYP/LANL2DZ method. Molecular mechanics computation was also conducted, and applicable parameter sets were determined.

\section{References}

[1] E. E. Kim, H. W. Wyckoff, J. Mol. Biol., 218, 449464 (1991).

[2] M. H. Le Du, T. Stigbrand, M. J. Taussig, A. Menez, E. A. Stura, J. Mol. Biol., 276, 9158-9165 (2001).

[3] S. K. Burley, P. R. David, A. Taylor, W. N. Lipscomb, Proc. Natl. Acad. Sci. USA, 87, 6878-6882 (1990).

[4] B. Chevrier, C. Schalk, H. Dorchymont, J. M. Rondeau, D. Moras, C. Tarnus,, Structure, 2, 283-291 (1994). 
[5] H. M. GreenBlatt, O. Almog, B. Maras, A. Spungin-Bialik, D. Barra, S. Blumberg, G. Shoham, J. Mol. Biol., 165, 620-636 (1997).

[6] D. Jozic, G. Bourenkow, H. Bartunik, H. Scholze, V. Dive, B. Henrich, R. Huber, W. Bode, K. Maskos, Structure, 10, 1097-1106 (2002).

[7] D. Jozic, J. T. Kaiser, R. Huber, W. Bode, K. Maskos, J. Mol. Biol., 332, 243-256 (2003).

[8] H. Sakiyama, R. Mochizuki, A. Sugawara, M. Sakamoto, Y. Nishida, M. Yamasaki, J. Chem. Soc. Dalton Trans., 1999, 997-1000.

[9] M. Head-Gordon, J. A. Pople, M. J. Frisch, Chem. Phys. Lett., 153, 503-506 (1988).

[10] M. J. Frisch, M. Head-Gordon, J. A. Pople, Chem. Phys. Lett., 166, 275-280 (1990).

[11] M. J. Frisch, M. Head-Gordon, J. A. Pople, Chem. Phys. Lett., 166, 281-289 (1990).

[12] M. Head-Gordon, T. Head-Gordon, Chem. Phys. Lett., 220, 122-128 (1994).

[13] S. Saebo, J. Almlof, Chem. Phys. Lett., 154, 83-89 (1989).

[14] W. Kohn, A. D. Becke, R. G. Parr, J. Phys. Chem., 100, 12974-12980 (1996).

[15] A. D. Becke, J. Chem. Soc., 98, 5648-5652 (1993).
[16] A. D. Becke, Phys. Rev. A, 38, 3098-3100 (1988).

[17] P. J. Hay, W. R. Wadt, J. Chem. Phys., 82, 270-283 (1985).

[18] J. J. P. Stewart, Stewart Computational Chemistry, 2007

http://openmopac.net/

[19] Norio Senda, 2001

http://winmostar.com/

[20] M. J. S. Dewar, E. G. Zeobisch, E. F. Healy, J. J. P. Stewart, J. Am. Chem. Soc., 107, 3902-3909 (1985).

[21] J. J. P. Stewart, J. Comp. Chem., 10, 209-220 (1989).

[22] J. J. P. Stewart, MOPAC2002 V1.5, Fujitsu Limited, Tokyo, Japan (2004).

[23] J. J. P. Stewart, J. Mol. Model., (doi:10.1007/s00894-007-0233-4).

[24] N. L. Alinger, J. Am. Chem. Soc., 99, 8127-8134 (1977).

[25] H. Goto, K. Ohta, T. Kamakura, S. Obata, N. Nakayama, T. Matsumoto, E. Osawa, CONFLEX, Conflex corp., Tokyo, Japan (2004).

[26] H. Sakiyama, K. Ono, T. Suzuki, K. Tone, T. Ueno, Y. Nishida, Inorg. Chem. Commun., 8, 372-374 (2005).

\section{Appendix 1}

Additional stretching parameters for MM2 methods

\begin{tabular}{lcc}
\hline Bond $^{a}$ & $k_{s} /(\mathrm{mdyn} / \AA)$ & $l_{0} / \AA$ \\
\hline 1-N(amine) & 5.000 & 1.446 \\
1-O(ether) & 5.360 & 1.402 \\
2-O(carboxylate) & 5.000 & 1.410 \\
2-O(phenoxo) & 5.000 & 1.410 \\
Zn-N(amine) & 5.000 & 2.100 \\
Zn-O(carboxylate) & 5.000 & 2.000 \\
Zn-O(phenoxo) & 5.000 & 2.000 \\
Zn-O(ether) & 5.000 & 2.200 \\
\hline
\end{tabular}

a 1: C(alkane, $\left.\mathrm{sp}^{3}\right), 2: \mathrm{C}\left(\right.$ alkene, $\left.\mathrm{sp}^{2}\right)$ 
Additional bending parameters for MM2 methods

\begin{tabular}{lcccc}
\hline Angle $^{a}$ & $k_{0} /\left(\operatorname{mdyn~} \AA / \mathrm{rad}^{2}\right)$ & $\theta_{0}\left(-\mathrm{XR}_{2}-\right)^{\circ}$ & $\theta_{0}(-\mathrm{XRH}-) /^{\circ}$ & $\theta_{0}\left(-\mathrm{XH}_{2}-\right)^{\circ}$ \\
\hline $1-1-\mathrm{N}($ amine $)$ & 0.500 & 109.471 & 109.471 & 109.471 \\
$1-1-\mathrm{O}($ ether $)$ & 0.500 & 109.471 & 109.471 & 109.471 \\
2-1-N(amine) & 0.500 & 109.471 & 109.471 & 109.471 \\
5-1-N(amine) & 0.500 & 109.471 & 109.471 & 109.471 \\
5-1-O(ether) & 0.500 & 109.471 & 109.471 & 109.471 \\
1-2-O(carboxylate) & 0.500 & 120.000 & 120.000 & 120.000 \\
2-2-O(phenoxo) & 0.500 & 120.000 & 120.000 & 120.000 \\
O(carboxylate)-2-O(carboxylate) & 0.500 & 120.000 & 120.000 & 120.000 \\
1-N(amine)-1 & 0.500 & 109.471 & 109.471 & 109.471 \\
1-N(amine)-Zn & 0.500 & 109.471 & 109.471 & 109.471 \\
2-O(carboxylate)-Zn & 0.500 & 120.000 & 120.000 & 120.000 \\
2-O(phenoxo)-Zn & 0.500 & 120.000 & 120.000 & 120.000 \\
Zn-O(phenoxo)-Zn & 0.500 & 120.000 & 120.000 & 120.000 \\
1-O(ether)-1 & 0.500 & 120.000 & 120.000 & 120.000 \\
1-O(ether)-Zn & 0.500 & 120.000 & 120.000 & 120.000 \\
\hline
\end{tabular}

a 1: C(alkane, $\left.\mathrm{sp}^{3}\right), 2$ : C(alkene, $\left.\mathrm{sp}^{2}\right), 5: \mathrm{H}$

Additional out-of-plane bending parameters for MM2 methods

\begin{tabular}{lc}
\hline Atoms $^{a}$ & $k_{s} /(\mathrm{mdyn} / \AA)$ \\
\hline 2-O(carboxylate $)$ & 0.050 \\
2-O(phenoxo) & 0.050 \\
\hline
\end{tabular}

a 2: C(alkene, $\mathrm{sp}^{2}$ )

Additional torsion parameters for MM2 methods

\begin{tabular}{lccc}
\hline Atoms $^{a}$ & $V_{1} /(\mathrm{kcal} / \mathrm{mol})$ & $V_{2} /(\mathrm{kcal} / \mathrm{mol})$ & $V_{3} /(\mathrm{kcal} / \mathrm{mol})$ \\
\hline $1-2-2-\mathrm{O}($ phenoxo $)$ & 0.000 & 10.000 & 0.000 \\
2-2-2-O(phenoxo) & 0.000 & 10.000 & 0.000 \\
1-2-O(carboxylate)-Zn & -0.930 & 8.000 & 0.000 \\
O(carboxylate)-2-O(carboxylate)-Zn & -0.930 & 8.000 & 0.000 \\
\hline
\end{tabular}

a 1: $\mathrm{C}\left(\right.$ alkane, $\left.\mathrm{sp}^{3}\right), 2: \mathrm{C}\left(\right.$ alkene, $\left.\mathrm{sp}^{2}\right)$

\section{Appendix 2}

Additional pseudo-potential parameters for MMFF94s method

\begin{tabular}{lcc}
\hline Bond Stretching & $k_{f} /\left(\mathrm{kcal} / \mathrm{mol} / \AA^{2}\right)$ & $l_{0} / \AA$ \\
\hline Zn-N(amine) & 500.0 & 2.100 \\
Zn-O(carboxylate) & 500.0 & 2.000 \\
Zn-O(phenoxo) & 500.0 & 2.000 \\
Zn-O(ether) & 500.0 & 2.200 \\
\hline
\end{tabular}

